\title{
Inappropriate Laughter in a Patient with Hypothalamic Hamartoma
}

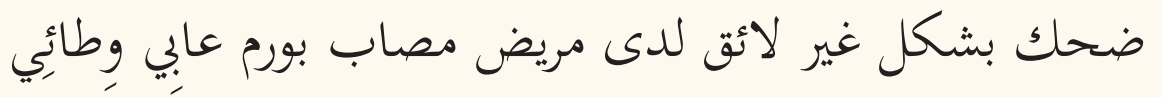

فارس الكلباني، فَراز أحمد، علاء المنزلاوي، آمنة الفطيسية

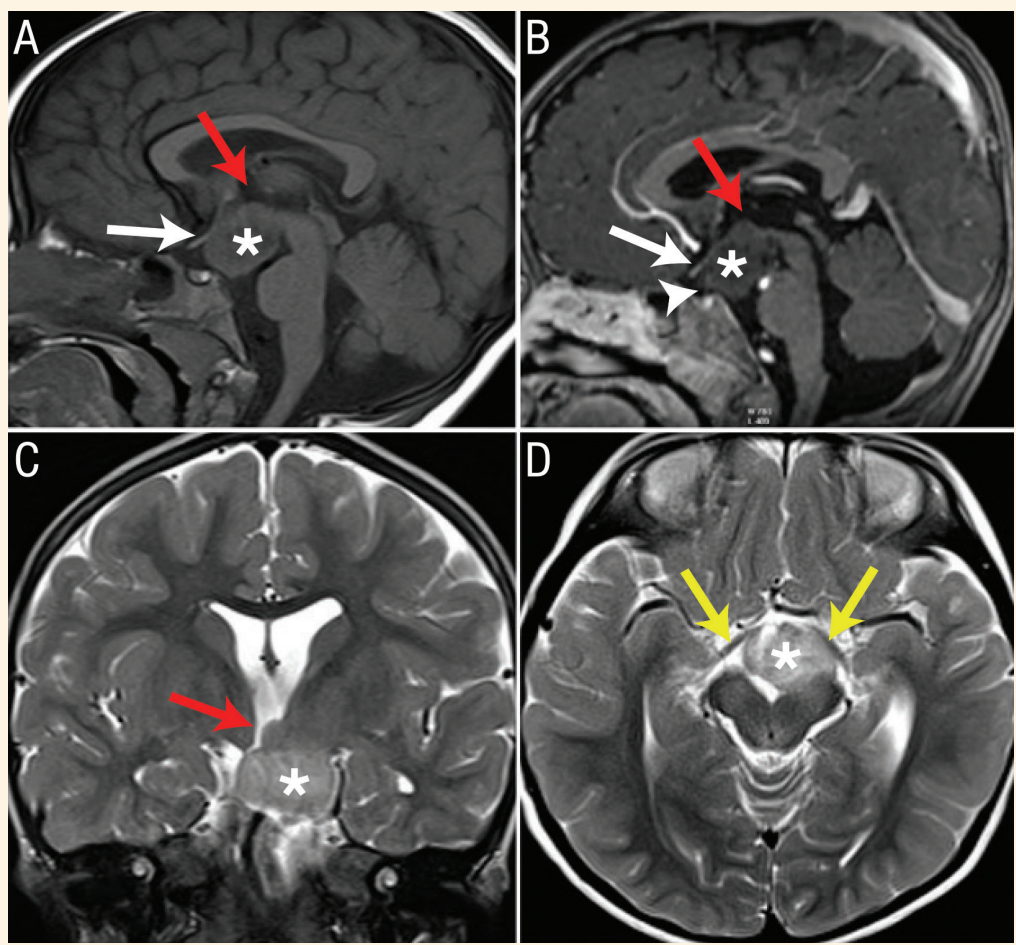

Figure 1: Sagittal T1-weighted magnetic resonance images (MRIs) of the brain of a two-year and seven-month-old boy before (A) and after (B) intravenous gadolinium contrast administration. They show a mass (asterisk) arising from the both above and below the floor of the third ventricle (red arrows). Accordingly, this mass has both vertical and horizontal planes of attachment to the floor of the third ventricle classifying it radiologically as Delalande type III. It occupies most of the suprasellar and interpeduncular cisterns. It compresses the optic chiasm (white arrows) and pituitary stalk (White arrowhead) anteriorly. Coronal (C) and axial (D) T2-weighted MRIs show the mass having a heterogenous hyperintense signal. It is seen stretching the optic tracts bilaterally (yellow arrows) in (D).

A TWO-YEAR AND SEVEN-MONTH-OLD BOY presented to the Emergency Department Muscat, Oman, in 2019, with a two-day history of uprolling of eyeballs, unresponsiveness and drooling of saliva lasting for seconds. There was no abnormal limb movement or loss of tone. The episodes were followed by a postictal state that lasted 30 minutes. There were no triggers for the episodes, such as fever or intercurrent illness. The patient was born at term gestation to a consanguineous marriage. Antenatal, birth and family history were unremarkable. His developmental milestones were appropriate for his age and his immunisation was up-to-date. A sleep electroencephalogram (EEG) showed abnormal interictal generalised epileptiform discharges with no associated clinical accompaniment. There were bilateral occipital epileptiform discharges with slowing over the left 


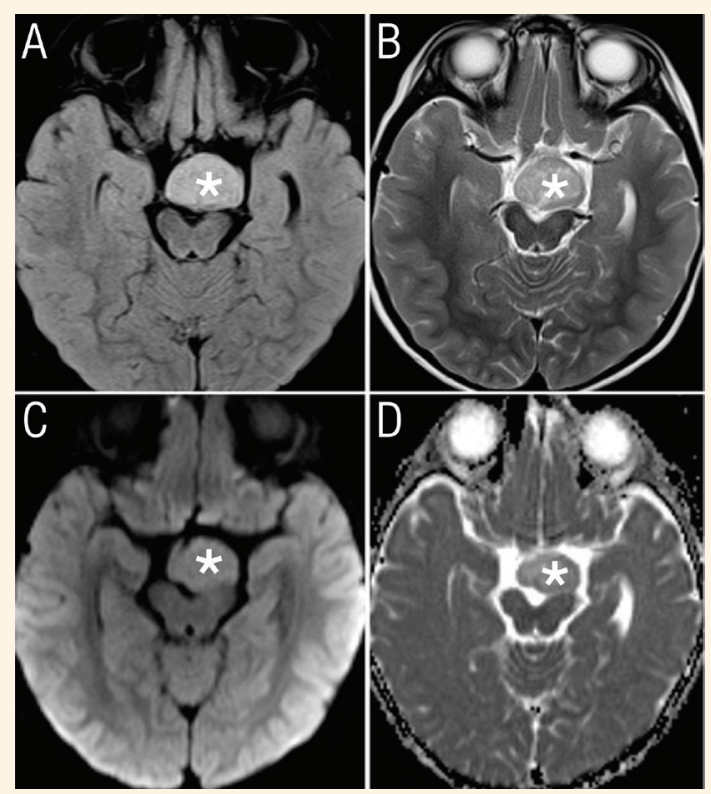

Figure 2: Magnetic resonance imaging (MRI) of the brain of a two-year and seven-month-old boy showing a hypothalamic (tuber cinereum) hamartoma (asterisk). It shows (A \& B) hyperintense signal on fluid-attenuated inversion recovery and T2 MRIs. Axial diffusionweighted MRI (C) and apparent diffusion coefficient map (D) images showing no evidence of diffusion restriction in this lesion.

posterior head region. In view of some lateralising features on EEG, MRI of the brain was done within three weeks of initial presentation, which revealed features of a hypothalamic hamartoma (HH; Figures 1 and 2). Formal visual field assessment was not possible due to the patient's young age. However, no visual symptoms were observed and endocrine workup was normal. Initial seizure control was achieved with levetiracetam reaching a dose of $40 \mathrm{mg} / \mathrm{kg} /$ day. Two months later, he developed multiple, sudden onset, unprovoked and uncontrollable laughing episodes that were occurring for no obvious reasons, suggestive of gelastic seizures. Postictally, he was immediately back to his baseline with no tiredness or sleepiness. Repeated routine EEG did not capture the events and showed no clear epileptiform abnormality except bilateral posterior slowing with left temporal dominance. Long-term video EEG was not performed. The episodes of gelastic seizures were difficult to control and required the administration of topiramate $(6 \mathrm{mg} /$ $\mathrm{kg} /$ day) as well as a short course of phenytoin $(6 \mathrm{mg} /$ $\mathrm{kg} /$ day) to control a cluster of focal seizures. Currently, he is on two anti-epileptic medications (levetiracetam and topiramate). Eight months later during follow-up, the father of the child reported mild hyperactivity. There was no evidence of neuroregression. During the write-up of this article, the patient was awaiting formal assessment with the developmental and behavioural paediatric team.
Although he was not yet seizure-free, the episodes were reasonably controlled with 1-2 brief daily episodes of eyelid twitching and occasional gelastic seizures once in 1-2 weeks. The parents were offered the option of surgical intervention, but decided against it.

\section{Comment}

As a rare developmental malformation, $\mathrm{HH}$ can display diverse clinical manifestations. It may present with intractable seizures, central precocious puberty and cognitive and neuropsychiatric disturbances. Seizures associated with $\mathrm{HH}$ can be of variable types and include focal seizures with impaired awareness, generalised tonic-clonic seizures, epileptic spasms, atonic, tonic, focal seizures with retained awareness and the hallmark gelastic seizures. ${ }^{1}$ Based on the radiological features, the $\mathrm{HH}$ of the current patient was classified as Delalande type III. ${ }^{2}$ The patient initially presented with focal seizure with impaired awareness and later developed gelastic seizures, which are a unique type of seizures that are usually seen in early childhood. Gelastic seizures are characterised by frequent brief attacks of inappropriate laughter. ${ }^{3}$ The EEG during a gelastic seizure is usually normal or may show non-specific abnormalities, which may contribute to the difficulty in establishing the diagnosis when gelastic seizures are the sole presentation. The seizures in $\mathrm{HH}$ are thought to be due to either the intrinsic epileptogenicity of the hamartoma itself or contributed by the extensive epileptogenic networks and possible kindling-like relationship between the $\mathrm{HH}$ and the cortex. ${ }^{4,5}$ This epileptogenic spread could possibly explain the EEG findings in the current patient.

Most patients with epilepsy secondary to $\mathrm{HH}$ develop an associated mood disorder, behavioural issues and/or cognitive delay. A significant proportion of patients with $\mathrm{HH}$ and epilepsy may present with central precocious puberty. However, growth hormone deficiency, hypothyroidism and adrenal insufficiency are rare. Although diabetes insipidus is not seen in non-operated $\mathrm{HH}$, it is frequently seen postoperatively. ${ }^{6}$

Treatment of $\mathrm{HH}$-associated epilepsy is challenging and complete seizure freedom with antiseizure medications is rarely achieved. Behavioural and learning difficulties as well as cognitive delay lead to an overall developmental impairment contributing to the increased morbidity associated with $\mathrm{HH}$. Treatment strategies in such cases of progressive encephalopathy associated with $\mathrm{HH}$ should be based on multidisciplinary care, including neurologists, 
neuropsychologists and neurosurgeons. Therapeutic options include anti-seizure medications, ketogenic diet therapy and vagal nerve stimulation. Neurosurgical options include microsurgical resection, endoscopic disconnection, stereotactic laser ablation therapy, gamma knife surgery and laser interstitial thermal therapies. Although surgical intervention remains the best treatment option for achieving seizure control in drug refractory cases, it is associated with a high risk of postoperative long-term endocrine dysfunction, especially with disconnection surgeries. Newer surgical techniques-including stereotactic radiofrequency thermo-coagulation, gamma knife surgery and laser interstitial thermal therapies-are reported to have better outcomes, though long-term follow-up of such patients is needed to monitor the neurodevelopmental and endocrine functions. ${ }^{7}$ Therefore, multidisciplinary care with regular follow up and monitoring is recommended in such cases to make timely decisions based on individual case progress and evolution. ${ }^{8}$

\section{References}

1. Parvizi J, Le S, Foster BL, Bourgeois B, Riviello JJ, Prenger E, et al. Gelastic epilepsy and hypothalamic hamartomas: Neuroanatomical analysis of brain lesions in 100 patients. Brain 2011; 134:2960-8. https://doi.org/10.1093/brain/awr235.

2. Delalande O, Fohlen M. Disconnecting surgical treatment of hypothalamic hamartoma in children and adults with refractory epilepsy and proposal of a new classification. Neurol Med Chir (Tokyo) 2003; 43:61-68. https://doi.org/10.2176/nmc.43.61.

3. Shahar E, Kramer U, Mahajnah M, Lerman-Sagie T, Goez R, Gross V, et al. Pediatric-onset gelastic seizures: Clinical data and outcome. Pediatr Neurol 2007; 37:29-34. https://doi.org/10.10 16/j.pediatrneurol.2007.03.003

4. Wu J, Gao M, Shen JX, Qiu SF, Kerrigan JF. Mechanisms of intrinsic epileptogenesis in human gelastic seizures with hypothalamic hamartoma. CNS Neurosci Ther 2015; 21:104-11. https://doi.org/10.1111/cns.12348.

5. Scholly J, Staack AM, Kahane P, Scavarda D, Régis J, Hirsch E, et al. Hypothalamic hamartoma: Epileptogenesis beyond the lesion? Epilepsia 2017; 58:32-40. https://doi.org/10.1111/epi.13755.

6. Harrison VS, Oatman O, Kerrigan JF. Hypothalamic hamartoma with epilepsy: Review of endocrine comorbidity. Epilepsia 2017; 58:50-59. https://doi.org/10.1111/epi.13756.

7. Arocho-Quinones EV, Koop J, Lew SM. Improvement of hypothalamic hamartoma-Related psychiatric disorder after stereotactic laser ablation: Case report and review of literature. World Neurosurg 2019; 122:680-3. https://doi.org/10.1016/j. wneu.2018.11.166.

8. Helen Cross J, Spoudeas H. Medical management and antiepileptic drugs in hypothalamic hamartoma. Epilepsia 2017; 58:16-21. https://doi.org/10.1111/epi.13758. 